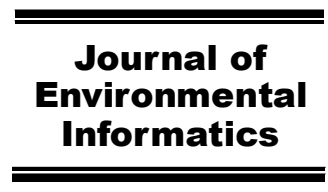

www.iseis.org/jei

\title{
A Study on the Performance of ARC Pavement for Traffic Noise Reduction - A SPB Comparison with Conventional Pavement
}

\author{
L. M. Dai ${ }^{1,2, *}$, Z. Lou $^{2}$, and A. Widger ${ }^{2}$ \\ ${ }^{I}$ Mathematics and Physics, North China Electric Power University, Beijing 102206, P.R. China \\ ${ }^{2}$ Industrial System Engineering, University of Regina, Regina, Saskatchewan S4S 0A2, Canada
}

Received 20 February 2007; revised 2 July 2007; accepted 28 January 2008; published online 2 September 2008

\begin{abstract}
This research investigates traffic noise reduction on highways paved with ARC material and establishes a quantitative model for evaluating the effect of traffic flow on traffic noise. A series of traffic noise measurements have been combined with traffic flow monitoring to compare the sound performance of ARC with conventional pavements and analyze the relationship between the noise levels over ARC pavement with traffic flow conditions. The 24-hour averaged Statistical Pass-By (SPB) noise levels indicate that the ARC pavement demonstrates better sound performance than that of conventional pavement in traffic noise reduction. The energetic averaging method is employed to study the relationship between traffic noise levels and traffic flow conditions. The applicability of ARC pavement to traffic noise reduction under various traffic flow conditions is also investigated. A relationship between traffic flow and traffic noise is established in terms of SPB noise levels and the traffic volumes for different vehicle categories. The relationship is employed to compare the noise reduction performance between ARC and conventional pavements under various traffic flow conditions. The research results indicate that ARC pavement is very effective at reducing the noise generated by large vehicles.
\end{abstract}

Keywords: ARC pavement, traffic noise reduction, traffic flow

\section{Introduction}

It is well known that noise can bring about many negative physiological and psychological effects for people. While many technologies have been developed to reduce industrial noise, traffic noise has become a major noise source for disturbing daily life of urban residents. Today, environmental pollution caused by traffic noise is very serious in some areas. In many cities, traffic noise levels on main streets can reach more than 80 dBA (Li et al., 2002; Ali and Tamura, 2003; Dai et al., 2005). However, the generally accepted traffic noise levels for residential areas should be less than $55 \mathrm{dBA}$ according to WHO (1999). It is recognized that a temporary shift in the threshold of hearing may occur at $L_{\text {Aeq }}$ levels higher than $80 \mathrm{dBA}$ (Sandberg and Ejsmont, 2002). As a result, traffic noise reduction activities are necessary in areas where the residents are seriously disturbed by traffic noise. Asphalt Rubber Concrete (ARC) pavement has shown better performance in traffic noise reduction, in comparison with that of conventional pavements. In many cases, construction of ARC pavement road is a better choice for reducing traffic noise in comparison with other methods, such as limitation of traffic flow

\footnotetext{
* Corresponding author. Tel.: +1 306585 4498; fax: +1 3065854855.

E-mail address: liming.dai@uregina.ca (L. M. Dai).
}

ISSN: 1726-2135 print/1684-8799 online

(C) 2008 ISEIS All rights reserved. doi:10.3808/jei.200800120 and construction of noise barriers.

ARC pavement was first developed in the middle of the last century. It has been proven with many applications around the world that this material has many advantages such as recycling discarded tire rubber, high water discharge ability, great durability with lower maintenance costs and good noise reduction (Bilawchuk, 2005). However, the acoustic performance of this pavement was not assessed until the early 1980s. Since then, many research activities have concluded that ARC pavement is capable of traffic noise reduction. According to data collected from other researchers, rubber asphalt pavement can, on average, reduce noise levels by 3 to $6 \mathrm{~dB}$ (Golebiewski et al., 2001; Fujiwara et al., 2004; Bilawchuk, 2005). In some cases, $10 \mathrm{~dB}$ noise level reductions can also be expected (Sacramento County Department of Environmental Review and Assessment and Bollard and Brennan, Inc, 2005; Rubber Asphalt Association).

Traffic noise is composed of two components. One is the sound wave generated directly from the noise source which includes mechanical sourced noise; engine and electric fan noise and noise generated by the interaction between tire and pavement. The second component is the noise reflected by the pavement surface. ARC pavement is a porous-surfaced pavement which has a larger capacity to absorb sound and reduce the reflected sound power and tire/pavement sourced sound power. In sum, the reduction in air pumping power, reduction in carcass vibration, changes in sound reflection geometry 
(Nelson, 1994), and increased sound power absorption (Ge and Wang, 2003) each contributes to an overall reduction in traffic noise on ARC pavement. Mechanical sourced noise has been reduced significantly with advancements in vehicle manufacturing technology, leaving tire/pavement borne noise to become the dominant noise in terms of passenger cars for speeds over $30 \mathrm{~km} / \mathrm{h}$ and transport trucks over $50 \mathrm{~km} / \mathrm{h}$ (Ulf Sandberg and Ejsmont, 2002).

Although great efforts have been given by the researchers and engineers on the traffic noise responses of ARC pavements since 1970's, however, a comprehensive understanding of the mechanisms of the traffic noise generation, propagation and reduction is far from reached, as the noise on ARC pavement is complex and involves numerous factors. A theoretically and practically sound methodology for quantifying the acoustic characteristics of ARC pavements with respect to the major traffic parameters has not been found in the current literature. A thorough and systematic investigation on the traffic noise behavior of ARC pavements is needed. This research intends to establish a quantitative methodology for evaluating the effects of the major factors such as traffic flow, types of vehicle, and noise energy on the traffic noise, on the basis of a thorough experimental investigation performed on test ARC and conventional roads. The acoustic response of ARC pavements is to be systematically studied, and the traffic noise on the ARC and conventional pavements is to be determined with a model established corresponding to given traffic parameters.

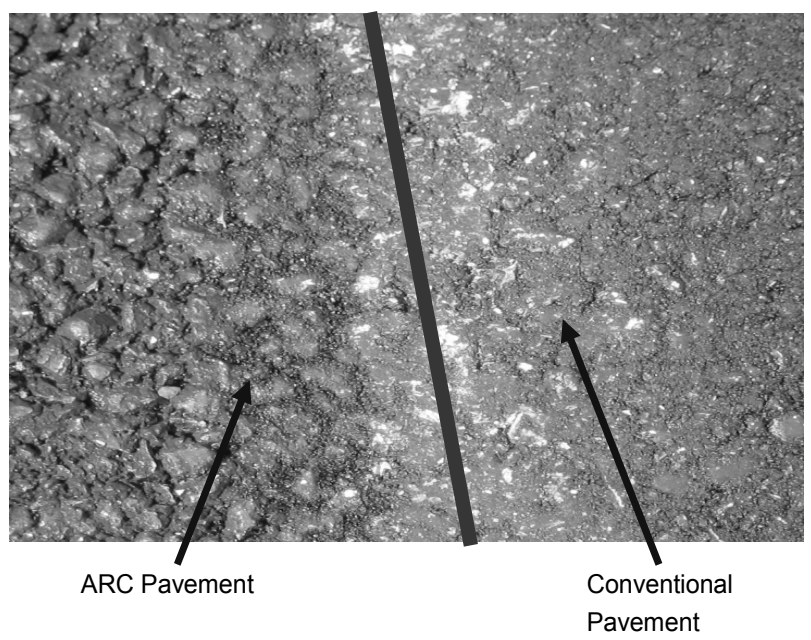

Figure 1. View of ARC and conventional pavement.

\section{Traffic Noise on the ARC Test Section}

\subsection{Road Test Methodology}

In August 2005, a test section of ARC pavement was paved on Highway Number 11 in Saskatchewan, Canada. This $10.3 \mathrm{~km}$ ARC test section is near Chamberlain, which is approximately $80 \mathrm{~km}$ from Regina, the capital of Saskatchewan. The test section includes four lanes; one driving lane and one passing lane in each direction (northbound and southbound). The driving and passing lanes in each direction were repaved.
However, only the driving lanes were repaved with ARC pavement where more than $80 \%$ of the passenger vehicles and more than $90 \%$ of the trucks travel. The southbound and northbound passing lanes were repaved with conventional asphalt concrete pavement. Before and after the road was repaved, a series of monitoring tests were preformed on the road to study the sound performance of the ARC pavement. A comparison of the two surfaces of ARC and conventional pavements is shown in Figure 1, where more open pores of the ARC pavement can be visualized with respect to the conventional pavement.

Traffic noise measurements and test methods can be classified into three groups; roadside measurements, on-board measurements and laboratory based measurements. Roadside measurements include the measurement and implementation of Statistical Pass-By (SPB), Controlled Pass-By (CPB) and Time-Averaged traffic noise methods. On-board measurements include the Close Proximity (CPX) and Close Proximity Sound Intensity (CPI) methods. These have all been implemented in previous traffic noise studies. In Japan, the time averaged method was employed to compare A-weighted sound pressure levels of a porous elastic road surface (PERS) with that of conventional road pavements (Fujiwara et al., 2004). The Time-Averaged method was performed with the SPB method to compare the sound performance of ARC and conventional pavements in Alberta, Canada (Bilawchuk, 2005). The CPB method was employed by some researchers to study the acoustic performance of dense asphalt and porous asphalt pavement in Poland (Golebiewski et al.; 2001). In sum, the Close Proximity Methods (CPB and CPI) focus on the study of noise generated by the interaction between tire and pavement, whereas the roadside measurements (SPB, CPB and Time-Average) can be employed to study the environmental influence of traffic noise. In the present research, the overall traffic noise including the tire/pavement noise and the other noise such as the noise of exhausting system, engine and carcass vibration are to be counted. The research also intends to investigate the environmental noise with as less as possible the other effects such as background noise, aerodynamic noise and reflection noise of the constructions in the vicinity of the test spot. Moreover, the maximum A-weighted sound pressure levels for large number of vehicle pass-bys are expected to be measured at a specified road-side location, together with the vehicle speeds. With these considerations, Time-Averaged traffic noise and Statistical Pass-By (SPB) methods were implemented for measuring the traffic noise for this research. ISO Standard 11819-1 and ISO Guidelines were adopted.

During the field tests, a Bruel and Kjær (B\&K) Observer 2260 sound intensity level meter was utilized for sound data acquisition. The B\&K 4198 microphone was used as the outdoor microphone with a $150 \mathrm{~mm}$ windscreen and a $1.5 \mathrm{~m}$ height tripod. Before each noise acquisition, the sound level meter was calibrated with B\&K Calibrator 4231 (Bruek and Kjær, 1995, 1998).

The "A" weighted network is known as to correlate well with the sensitivity of human hearing and has been widely accepted as a sound level parameter in noise studies. In practice, 
the so called "equivalent traffic noise levels" are collected in the form of "A" weighted Equivalent Continuous Sound Levels $\left(L_{A e q}\right)$ and this practice was adopted in the present research. In the field the noise was measured in the form of $1 / 3$ Octave frequency bands and the overall frequency broadband noise data were collected. The time-weighting on the 2260 observer is set at " $F$ " (fast) during the tests. The A-weighted sound and noise levels are recorded as a 30 -second Equivalent Continuous Sound Level $\left(L_{A e q 30}\right)$ for proper data collection and storage.

Noise tests were performed in June 2005 before the test strip was resurfaced with ARC pavement and the tests were repeated in September 2005 after the resurfacing. Traffic noise measurements were recorded on weekdays only, for the sake of consistency, over a 24-hour period. In reference to the traffic noise test standards, the tests were performed under good weather conditions, no rain, no wind or only light winds and at a location absent of noise sources other than traffic and with no obvious sound barrier or reflecting objects nearby. According to the ISO 11819-1 standard, the test microphone should be located 7.5 meters from the centerline of the road. However, in consideration of highway traffic safety and to diminish the impact to free traffic flow, two outdoor microphones were set up 15 meters from the centerline of the nearest driving lane on both sides of the road as illustrated in Figure 2. Traffic noise levels were measured simultaneously in both directions during the tests; northbound and southbound.

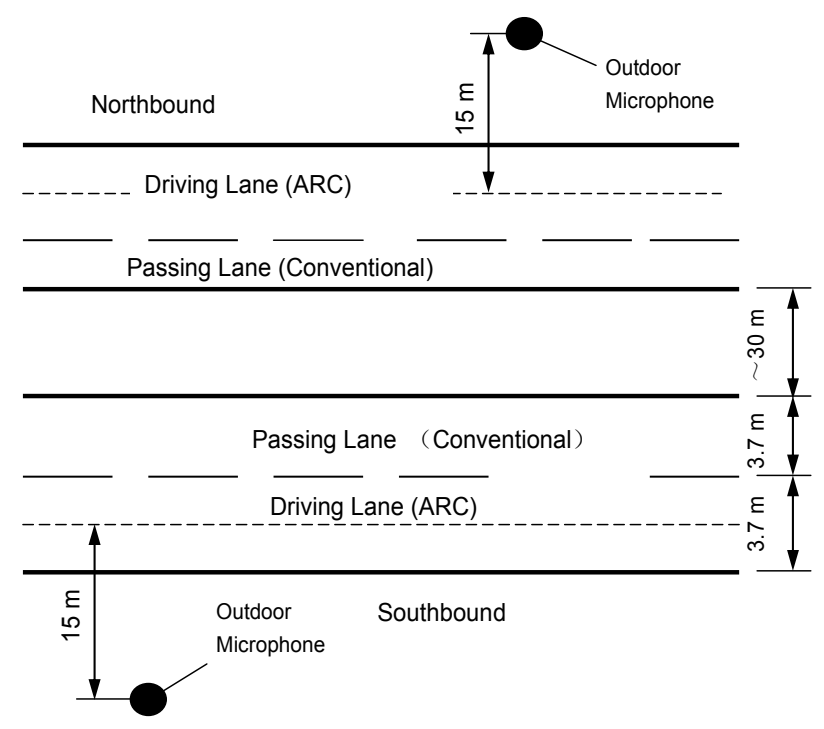

Figure 2. Layout of microphones.

During the road test, the maximum sound power from representative vehicles passing by the sound meters were recorded as Statistical Pass-By (SPB) noise levels. Representative vehicles were selected during normal driving conditions without acceleration or deceleration and having enough distance from other vehicles and the recorded noise levels were not influenced by other noise sources. The vehicles are classified into three categories (light vehicles, medium trucks and large trucks) in accordance with Federal Highway Administration (1998) vehicle classifications -- categories 2 and 3, categories 4 through, 10 and categories 11 through 14, respecttively.

Sound pressures were recorded and calculated automatically by the sound level meter's software as $L_{\text {Aeq30 }}$. The TimeAveraged traffic noise levels $L_{A e q T}$ were calculated with the following equation:

$$
L_{\text {AeqT }}=10 \log \left(\frac{t_{0}}{T} \sum_{i=1}^{n} 10^{L_{\text {Aeq } 30} / 10}\right), t_{0}=30 \mathrm{~s}
$$

where $T$ is the elapsed time, $t_{0}$ is the reference time, and $n$ is the number of intervals in an elapsed time.

The category and speed of each vehicle were collected automatically using equipment installed on Highway 11 near the test spots by Saskatchewan Highways and Transportation.

\subsection{Analysis of the Road Test Results}

\subsubsection{The 24-hour Time-Averaged Traffic Noise Level}

The 24-hour Time-Averaged traffic noise levels can be used to reasonably represent the correlation of community responses (Dai et al., 2005). Two sets of continuous 24-hour equivalent traffic noise data were used for the Time-Averaged noise level study. One set of data were acquried from 3:00 a.m. June $27^{\text {th }}$ to $3: 00$ a.m. June $28^{\text {th }}, 2005$ before the ARC pavement was repaved. The other set was acquired from 8:00 a.m. September $19^{\text {th }}$ to $8: 00$ am of September $20^{\text {th }}, 2005$ after the ARC pavement was paved. The results of the 24-hour TimeAveraged noise measurements for the northbound and southbound roads are presented in Table 1 . As can be seen in Table 1, ARC pavement material has a significant effect on traffic noise reduction in comparison with noise data measured over conventional pavement, before the ARC pavement was paved. Table 1 shows that northbound traffic sound levels were reduced by $4.1 \mathrm{~dB}$ and the southbound traffic sound levels were reduced by $4.9 \mathrm{~dB}$ after the road was resurfaced. This noise reduction is very significant.

The decibel sound level is described by the amplitude of the pressure fluctuations, which can be defined by the following equation (Bruel and Kjaer, 2000):

$$
L_{p}=10 \log _{10}\left(p^{2} / p_{r e f}{ }^{2}\right)
$$

where $L_{p}$ represents the logarithmic sound power scale, $p$ represents the sound pressure and $p_{\text {ref }}$ represents the reference sound pressure $\left(20 \times 10^{-6} \mathrm{~Pa}\right.$ for air $)$.

Noise reflects the energy of sound fluctuations in the air (Bruek and Kjær, 2000) and the sound energy scale is described by the amplitude of the pressure fluctuations. Based on Equation (2), a 4-dB decrease in sound level $\left(L_{p}\right)$ reduces the sound pressure $(p)$ by more than $50 \%$. This implies a significant amount of sound energy is actually reduced. 
Table 1. The 24-Hour's Time-Averaged Traffic Noise Level

\begin{tabular}{llll}
\hline Bound & Conv. $^{*}$ & ARC $^{* *}$ & Difference (After-Before) (dBA) \\
\hline North & 67.6 & 63.5 & -4.1 \\
South & 66.9 & 62.0 & -4.9 \\
\hline
\end{tabular}

$* 3: 00$ June $27^{\text {th }}$ to $28^{\text {th }}, 2005 ; * * 8: 00$ September $19^{\text {th }}$ to $20^{\text {th }}, 2005$

The 24-hour traffic volume curves from the northbound and southbound roads are presented in Figures 3 and 4 respectively. Figure 3 shows the traffic volume measured on the test road with conventional pavement, before ARC resurfacing and Figure 4 graphs the traffic volume over the ARC pavement. From the two figures, it can be observed that traffic volumes are almost the same before and after ARC resurfacing. The similarities in traffic volume over conventional and ARC pavement are needed for a reliable study on traffic noise over roads with different pavements.

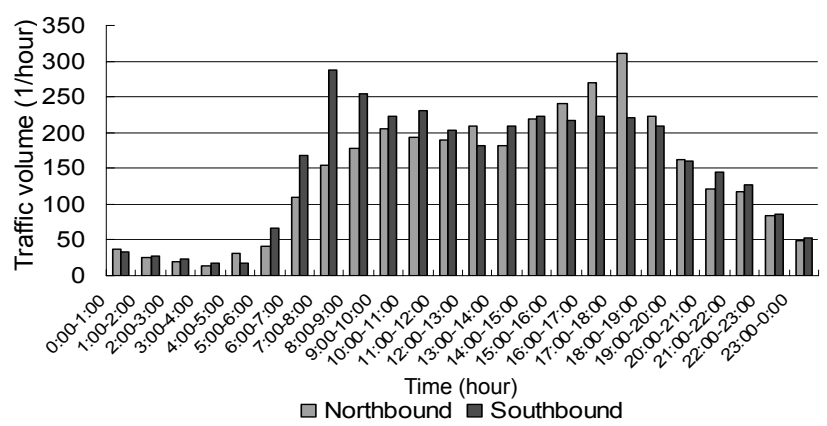

Figure 3. Traffic volume before road was repaved.

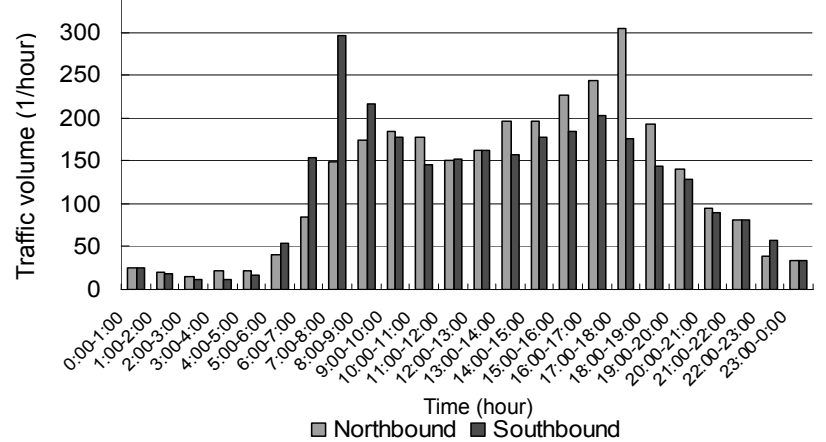

Figure 4. Traffic volume after road was repaved.

Figures 5 and 6 show 24-hour noise level curves for the northbound and southbound roads, respectively. The noise level of the test section reaches its highest value during the day and drops to its lowest value in the morning a few hours before dawn over both conventional and ARC pavements. The noise levels also reflect the traffic volume of Highway 11 (shown in Figures 3 and 4); the traffic volume peaks during the day and drops to a minimum before dawn. This indicates that the volume of traffic flow has a significant effect on traffic noise on both conventional and ARC pavements. Despite the dramatic changes in traffic volume, ARC pavement shows a significant correlation with noise reduction in comparison with conventional pavement. As shown in the figures, more than $3 \mathrm{dBA}$ of the day-time noise reduction can be observed. The 24-hour noise level and traffic volume results show that when traffic volume reaches a certain point, the ARC pavement could present better noise reduction performance in comparison with conventional pavement. A very low traffic volume is required to show that ARC pavement can achieve improved traffic noise reduction. When hourly traffic volumes on each side of road are higher than 50, the 1-hour equivalent $\left(L_{\text {AeqlH }}\right)$ noise reduction level of ARC pavement can be as high as 4 to $6 \mathrm{dBA}$. However, noise reduction levels do not change significantly even though the traffic volume changes dramatically.

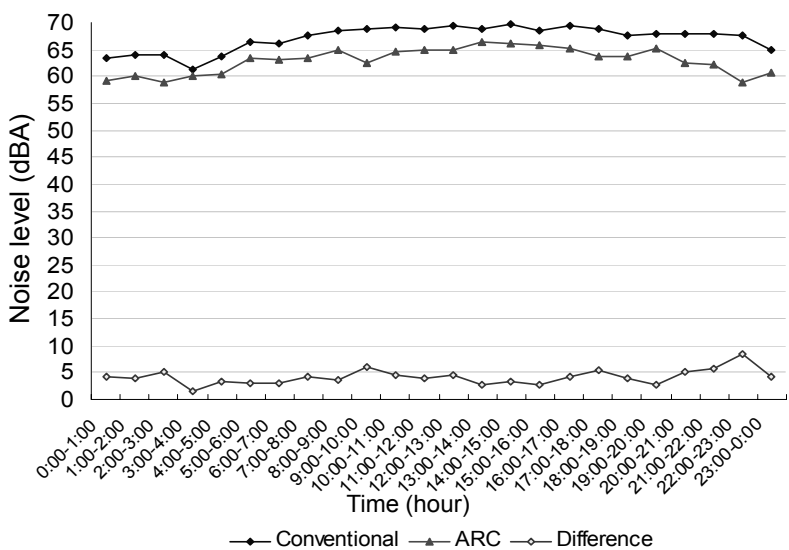

Figure 5. 24-hour noise level curve of northbound.

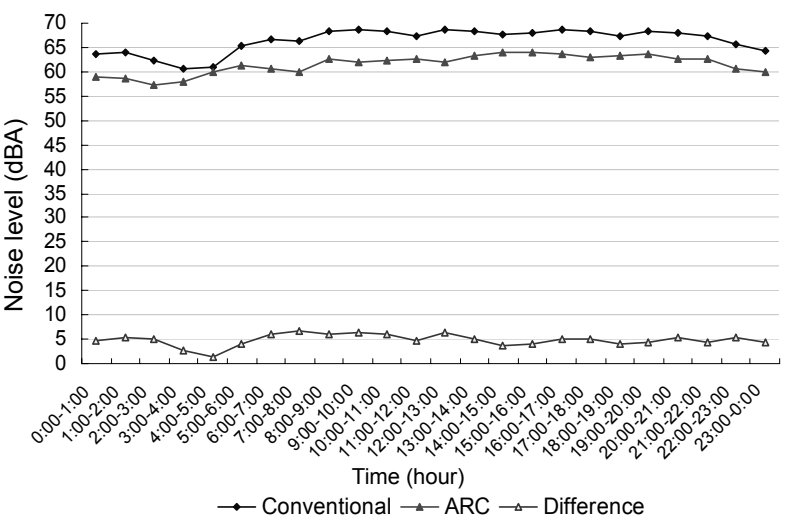

Figure 6. 24-hour noise level curve of southbound.

Not only are the overall spectral sound levels significant, as discussed above, the spectral content of the traffic noise is also important for traffic noise analysis. Figures 7 and 8 show the 24-hour Spectral Noise Measurements on Highway 11 for the northbound and southbound roads, respectively. The spectral sound levels over ARC pavement are higher or not significantly lower than that of conventional pavement when the frequency of the noise is $500 \mathrm{~Hz}$ or lower. This frequency area is mainly affected by wind and the engine noise of heavy trucks (Ruhala, 1999). Wind turbulence contributed to the slightly higher noise levels at lower frequencies over ARC pavement because the wind was stronger than that over conventional 
pavement. A significant sound level reduction is shown in the frequency range between $630 \mathrm{~Hz}$ and $6.3 \mathrm{k} \mathrm{Hz}$ for noise levels measured over ARC pavement. This frequency range is due mainly to tire/pavement noise. Recognition of this is significant, since human hearing is most sensitive to frequencies between $2 \mathrm{kHz}$ and $5 \mathrm{kHz}$ (Bruel and Kjaer, 2000). Therefore, sound level reduction in this range is most desirable and effective for traffic noise control.

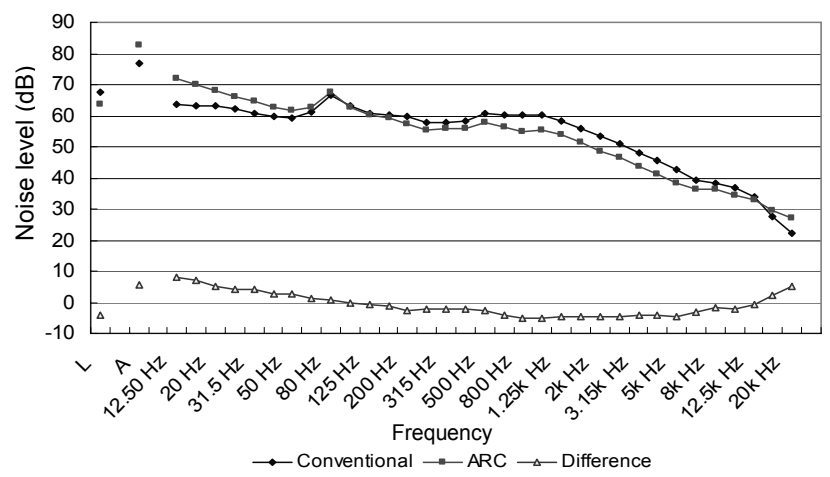

Figure 7. 24-hour spectral noise monitoring results at highway 11 northbound.

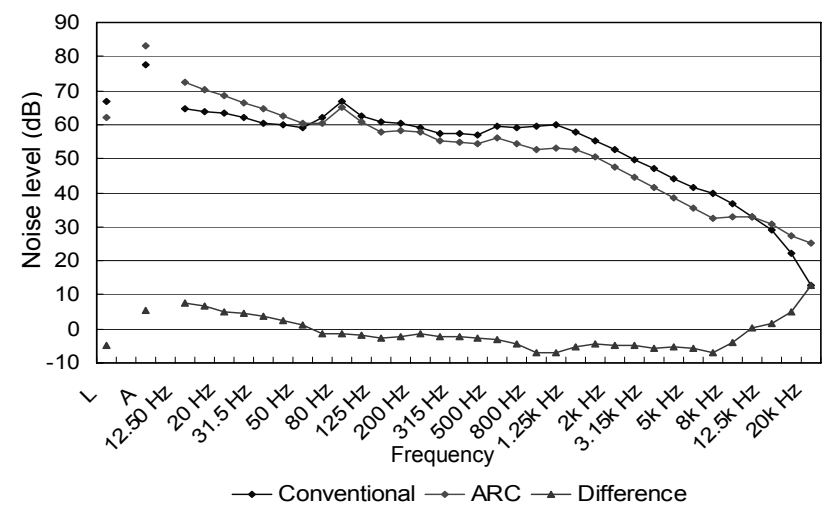

Figure 8. 24-hour spectral noise monitoring results at highway 11 southbound.

\subsubsection{Statistical Pass-By Noise Level}

The purpose of employing the Statistical Pass-By (SPB) method is to collect the maximum sound power of each vehicle passing by the measurement spot, so that a statistical index of noise levels for particular vehicle types can be acquired. The SPB method can be employed to account for the acoustic performance of individual vehicles. By comparing SPB noise levels over different pavements, this method could be used to study the influence of pavement conditions on environmental noise. Table 2 shows the Statistical Pass-By noise level results. The noise reduction of light autos, medium trucks and large trucks over ARC pavement with respect to conventional pavement is $5.5 \mathrm{~dB}, 5.4 \mathrm{~dB}$ and $6.3 \mathrm{~dB}$, respectively. In the table, "Conv" stands for conventional pavement, "Ave" represents the average A-weighted value, "Std Dev" represents standard deviation, "Before-After" designates the difference in $\mathrm{dBA}$ for traffic noise measured before and after the ARC pavement was applied. The results indicate that ARC pavement has a significant effect on noise reduction for all kinds of vehicles.

The following can be concluded from the noise and traffic volume data obtained and the analysis described above:

1) ARC pavement has better noise reduction performance in comparison to that of conventional pavement in the frequency range where human hearing is most sensitive.

2) The traffic volume analysis shows that traffic noise levels are directly related to the traffic volume and vehicle type.

3) ARC pavement can be used to reduce traffic noise levels for most traffic volume conditions.

4) ARC pavement has the ability to reduce traffic noise for all three types of vehicles tested in the research and the noise level reduction is significant.

Table 2. Statistical Pass-By Noise Level (Ave.dBA)

\begin{tabular}{|c|c|c|c|c|c|}
\hline Class & Conv. & $\begin{array}{l}\text { Std } \\
\text { Dev }\end{array}$ & ARC & $\begin{array}{l}\text { Std } \\
\text { Dev }\end{array}$ & $\begin{array}{l}\text { Difference } \\
\text { Before-After }\end{array}$ \\
\hline Light Autos (I) & 76.2 & 1.4 & 70.7 & 1.4 & -5.5 \\
\hline $\begin{array}{l}\text { Large trucks, } \\
\text { single-axel (II) }\end{array}$ & 86.3 & 1.4 & 80.9 & 1.6 & -5.4 \\
\hline $\begin{array}{l}\text { Large trucks, } \\
\text { multi-axel (III) }\end{array}$ & 87.8 & 1.2 & 81.5 & 1.8 & -6.3 \\
\hline
\end{tabular}

\section{Relationship between Traffic Flow Conditions and Traffic Noise Levels on Conventional and ARC Pavements}

\subsection{Traffic Noise Level Simulation Model Analysis}

It is crucial to establish a relationship between traffic noise levels and traffic flow conditions such as traffic volume, vehicle composition and pavement conditions, to evaluate the impact of traffic noise on the vicinities surrounding the road. Establishing this relationship is also important for assessing the acoustic performance of the road pavement. Additionally, implementation of this relationship may provide government agencies and interested private sectors to control and assess the traffic noise under given traffic conditions.

Although many factors affecting traffic noise are taken into account in traffic noise prediction models in the market and literature, a model or a software package suitable for predicting traffic noise over ARC pavement road cannot be found. The Federal Highway Administration Traffic Noise Prediction Model (TNM) (Federal Highway Administration, 1998) is one of the most widely used traffic noise assessment models in North America. This model can only be applied for traffic noise prediction on five kinds of pavement. They are DenseGraded Asphaltic Concrete (DGAC), Portland Cement Concrete (PCC), Open-Graded Asphaltic Concrete (OGAC) and a composite pavement type consisting of data for DGAC and PCC combined. However, ARC pavement is not included.

Based on data collected from the road tests on Highway 11 , the ARC pavement produces a noise reduction of 4 to 6 $\mathrm{dB}$ during most times of the day, regardless of the significant 
changes in traffic volume or vehicle composition, as illustrated in Figures 5 and 6 . While considering the advantages of ARC pavement for noise reduction, a new model for quantitatively predicting and analyzing the traffic noise on ARC pavement needs to be developed. This is one of the main goals of the present research.

In establishing the model for the traffic noise prediction, the following points need to be considered:

1) The traffic noise levels and traffic flow data, measured on Highway 11 in Saskatchewan, Canada, show that volume of traffic has a significant effect on traffic noise, as shown in Figures 3, 4, 5 and 6. Traffic volume is easier to monitor in comparison with the other factors that effect traffic noise levels. Therefore, traffic volume should be a main factor to be considered in establishing the noise prediction model.

2) Periodicity of traffic volume can be found on many highway and city roads. This periodicity depends on the hour and day of the week. Using traffic volume as a main factor for noise level prediction provides advantages for predicting traffic noise once the periodicity is found.

3) The SPB noise level is a parameter usually employed for traffic noise studies. Compared to long-term noise monitoring data, which is usually affected by other noise sources, SPB data is more accurate since it is measured right at the moment the vehicle passes. The measurement process and the effect of other noise sources is easy to control. Thus, to assess traffic noise levels by means of establishing the model, SPB noise levels should be taken into account.

4) SPB noise levels are also reliant on the type of vehicle on the road. Therefore, the corresponding category of vehicle should be specified in utilizing SPB levels.

Based on the above discussion, in developing the desired model for evaluating the relationship between traffic flow and traffic noise levels, the traffic volume and SPB noise levels of certain vehicle categories are counted in this research.

\subsection{Energetic Averaging Method for Calculating Equiva- lent Noise Level}

The energetic averaging method has been shown to be efficient in acoustic analysis (Sandberg and Ejsmont, 2002). With this method, the logarithmic sound level $(\mathrm{dB})$ is recalculated to the corresponding linear sound intensity (represented by energy). All the linear values are averaged arithmetically and, finally, one calculates back to the logarithmic level. Therefore, this method is implemented to develop the desired model. The implementation of the energetic averaging method for simulating traffic noise levels on the highway is based on the following conditions:

1) Traffic noise is the dominant source of noise on the roads. Background noise and other noise sources are much lower than the noise emitted by vehicles. Thus, the noise levels emitted by sources other than traffic noise can be ignored.

2) There are no obvious obstacles and sound absorbing objects near the spot where measurements are made. Thus, the traffic noise energy may not be strengthened or weakened by objects within the vicinity of the road.

3) Vehicle speed is an important parameter that influences traffic noise (Sandberg and Ejsmont, 2002). Usually traffic noise increases as vehicle speed increases. However, the speed of the vehicles on the highway is relatively stable and nearly constant on the highway. Therefore, the variation of the vehicle speed is not considered in this research, though it can be considered with the model to be established.

4) Vehicles moving on the highway are divided into three categories in this research. For a specified vehicle category, the sound energy emitted by a vehicle can be represented by the averaged energy over all the vehicles involved in this category, provided that the speeds of the vehicles does not vary significantly. Therefore, the average energy in a specified category is used to calculate the energy of the vehicles involved.

If the conditions mentioned above can be satisfied, the roadside noise levels can be calculated by using the following process.

Equivalent sound levels are a useful measure of traffic noise. According to Equation (2), the logarithmic sound pressure $L_{p}$ is calculated from the sound pressure $p$. The equivalent sound level is a constant sound level over a given time interval which has the same energy within the actual measured time duration:

$L_{e q}=10 \log \left(\frac{t_{0}}{T} \int \frac{p(t)^{2}}{p_{\text {ref }}^{2}} d t\right)$

where $L_{e q}$ is the equivalent sound level, $t_{0}$ represents the reference time, $t_{0}=1 \mathrm{~s}$ and $T$ represents the objective time interval.

In considering the energy involved, sound energy can be used to replace the sound pressure. Thus, calculating the $L_{e q}$ by means of sound intensity is much simpler than by means of sound pressure. Sound energy is usually expressed in the form of sound intensity $E$ :

$E=\int_{-\infty}^{+\infty} \frac{p(t)^{2}}{p_{r e f}^{2}} d t$

The average sound intensity over a given time interval can be represented by the average sound energy generated by all vehicles passing by the test spot:

$E=\frac{t_{0}}{T} \sum_{i=1}^{n} e_{i}$

where $e_{i}$ represents the sound intensity over a given time interval.

For TNM model vehicle classification, the vehicles are classified into 5 categories: automobiles ( 2 axles and 4 tires), medium trucks ( 2 axles and 6 tires), heavy trucks ( 3 or more 
axles and 6 or more tires), buses (2 or 3 axles and 6 or more tires) and motorcycles ( 2 or 3 tires). This classification method basically covers all kinds of vehicles appearing regularly on North American roads. Since our research focuses on the ARC pavement of Highway 11 -- the main road connecting Regina and Saskatoon, there are very few buses or motorcycles and the number of buses and motorcycles is limited in comparison to other types of vehicles. Therefore, three kinds of vehicles are considered in the present research, they are light vehicles (Class I), medium trucks (Class II) and large trucks (Class III). For the highway section considered, the average speed of a particular kind of vehicle does not fluctuate significantly. This can be seen from the low standard deviation values for the traffic noise levels corresponding to all three kinds of vehicles (Table 2), as the sound intensities for a given category of vehicles are not significantly different. This implies that the average sound intensity can be employed to represent the sound intensities for a given category of vehicle. The traffic sound intensity of a test spot along the highway is then expressed as the combination of the three vehicle types as shown below:

$E=\frac{t_{0}}{T} \sum_{j=1}^{3}\left(m_{j} e_{j}\right)$

where $m_{j}$ represents the volume of a particular vehicle type and $e_{j}$ represents the average sound intensity energy of the particular vehicle type:

$e_{j}=10^{L E_{j}}$

where $L E_{j}$ represents the average sound exposure intensity level of a particular vehicle.

Since the highway consists of two lanes in each of the two directions, the noise data acquired for a lane in one direction may be affected by the noise generated by the traffic from the other lanes in the opposite direction. As is well known, the noise level decreases as the distance between the source and receiver increases. In the reference TNM Technical Manual, the relationship between traffic noise intensity and distance can be considered as linear in the perpendicular direction. Based on this principle, the sound intensity of the test spot can be given by:

$E_{(\text {total })}=\left[\frac{d_{0}}{d_{\text {near }}} E_{(\text {near })}+\frac{d_{0}}{d_{\text {far }}} E_{(\text {far })}\right]$

where $d_{0}$ represents the reference distance between the middle line of the traffic lanes and the SPB sound level test spot (m), $d_{\text {near }}$ represents the perpendicular distance between the centerline traffic lane of the nearest side $(\mathrm{m})$ and $d_{\text {near }}$ represents the perpendicular distance between the centerline traffic lane of the furthest side (m).

A combination of Equations (3) and (8) gives the equiva- lent traffic sound level at the test spot:

$$
\begin{aligned}
L_{\text {Aeq }}= & 10 \log \left\{\frac { t _ { 0 } } { T } \left[\sum_{j=1}^{3} m_{(\text {near }) j} \frac{d_{0}}{d_{\text {near }}} 10^{L E(\text { (near })_{j} / 10}\right.\right. \\
& \left.\left.+\sum_{j=1}^{3} m_{(\text {far })_{j}} \frac{d_{0}}{d_{\text {far }}} 10^{L E(\text { far })_{j} / 10}\right]\right\}
\end{aligned}
$$

where $m_{(\text {near })}$ and $m_{(f a r)}$ represent the traffic volume of the near and far sides, respectively; $L E_{(\text {near })}$ and $L E_{(f a r)}$ represent the average sound intensity of the near and far sides, respectively.

This is an expression describing the noise level for a given traffic volume. This equation provides a relationship between traffic flow and traffic noise level and can be used to evaluate the noise reducing characteristics of ARC pavement.

Since the SPB noise and traffic volume data are easily measured along Canadian highways, this equation can be extended as a quick traffic noise assessment for other projects conducted in Canada or North America.

\subsection{Numerical Simulation for Traffic Noise Levels of Con- ventional and ARC Pavement}

The average SPB noise level data acquired from the road tests (Table 2) were recalculated as sound intensity, according to Equation (7) and used as a reference sound intensity for each kind of vehicle. Referring to many traffic noise assessment standards, the 1-hour equivalent traffic noise level -$L_{A e q I H}$ is adopted as a traffic noise evaluation value in our simulation. Thus, the time interval based on Equation (9), $T$ equals $3600 \mathrm{~s}$. According to the horizontal geometric dimensions of the highway, the distance between the spot where the noise data is acquired and the center line of the driving lane at the near and far sides is 15 and $60 \mathrm{~m}$, respectively. The traffic volume was measured as a function of vehicle type and was provided by the Saskatchewan Highways and Transportation Department. With the data collected, the 1-hour equivalent traffic noise level $\left(L_{A e q 1 H}\right)$ can be calculated using Equation (9).

Table 3. Paired t-test Results of the Noise Level on Conventional Pavement (before Repaved)

\begin{tabular}{lll}
\hline Statistical values & Predicted $\mathrm{L}_{\mathrm{eqA}}(\mathrm{dB})$ & Measured $\mathrm{L}_{\mathrm{eqA}}(\mathrm{dB})$ \\
\hline Mean & 66.19 & 66.13 \\
Known variance & 2.84 & 2.65 \\
Observation & 86 & 86 \\
\hline Pearson correlation $(r)$ & 0.99 \\
Hypothesized mean difference $\left(H_{0}\right)$ & 0 \\
Significant level $(\alpha)$ & 0.05 \\
Freedom degree & 85 \\
Paired t-test & 0.87 \\
Probability two-tail & 0.38 \\
t Critical two-tail & 1.99 \\
\hline
\end{tabular}

The paired t-test technique (Pamanikabud et al., 2002) is used to test the fit of the simulated and measured noise levels. 
For conventional pavement, 86 sets of data were collected before the highway was repaved. Tables 3 and 4 show the paired t-test results of the simulated and measured noise levels over conventional and ARC pavements. Figures 9 and 10 show the dimensional test of the predicted and measured noise levels over conventional and ARC pavements, respectively.

Table 4. Paired t-test Results of the Noise Level on ARC Pavement (after Repaved)

\begin{tabular}{lll}
\hline Statistical values & Predicted $\mathrm{L}_{\mathrm{eqA}}(\mathrm{dB})$ & Measured $\mathrm{L}_{\mathrm{eqA}}(\mathrm{dB})$ \\
\hline Mean & 61.91 & 61.98 \\
Known variance & 2.74 & 2.98 \\
Observation & 68 & 68 \\
\hline Pearson correlation $(r)$ & 0.90 \\
Hypothesized mean difference $\left(H_{0}\right)$ & 0 \\
Significant level $(\alpha)$ & 0.05 \\
Freedom degree & 67 \\
Paired t-test & -0.50 \\
Probability two-tail & 0.62 \\
t Critical two-tail & 2.00 \\
\hline
\end{tabular}

Paired t-tests, adopt a significance level of 0.05 . The calculated t-test values for conventional and ARC pavements are 0.87 and -0.50 , respectively. The t-test values from the conventional and ARC pavements are less than 1.99 - the critical value at the 0.05 significance level. This result implies that the simulated noise levels for the two pavements fits well with the measured values. The Pearson correlation (r) reaches 0.99 and 0.90 for conventional and ARC pavements which shows sufficient linearity for the overall noise level interval and implies the simulation fits all traffic flow conditions -- from lower to higher vehicle volume density.

The dimension test adopts a $45^{\circ}$ line $(x=y)$ for the datum line, which represents $100 \%$ confidence that the simulated results fit the measured results. In Figures 9 and 10, all of the 86 sets of data $(100 \%)$ from the conventional pavement and 66 of the 68 sets of data $(97.1 \%)$ from ARC fit with the simulation data at a $95 \%$ level of confidence.

Traffic noise level simulations using the energetic averaging method indicate that time equivalent noise level reduction over ARC pavement is due to the noise reduction in SPB noise level for a particular vehicle. Our results show that all types of vehicles considered in this research have a lower noise level over ARC pavement than over conventional pavement. Therefore, ARC pavement can be used to reduce noise levels where the traffic noise is the dominant form of environmental noise. Moreover, ARC pavement can be applied to a road with a wide variety of traffic conditions, such as different volume densities and vehicle compositions, to achieve noise reduction.

\section{Discussions}

It is significant to study the traffic noise reduction ability of ARC pavement with different traffic flow compositions to assess the applicability of this pavement's sound performance.
The difference in noise intensity for traffics over conventional and ARC pavement under the same traffic flow conditions can be expressed by:

$e_{(d i f f)}=\frac{t_{0}}{T} \sum_{j=1}^{3} m_{j}\left(10^{L E_{(\text {conv })}}-10^{L E_{(A R C)}}\right)$

where $L E_{(\text {covn })}$ and $L E_{(A R C)}$ represent the average sound intensity for certain vehicle types on conventional and ARC roads, respectively.

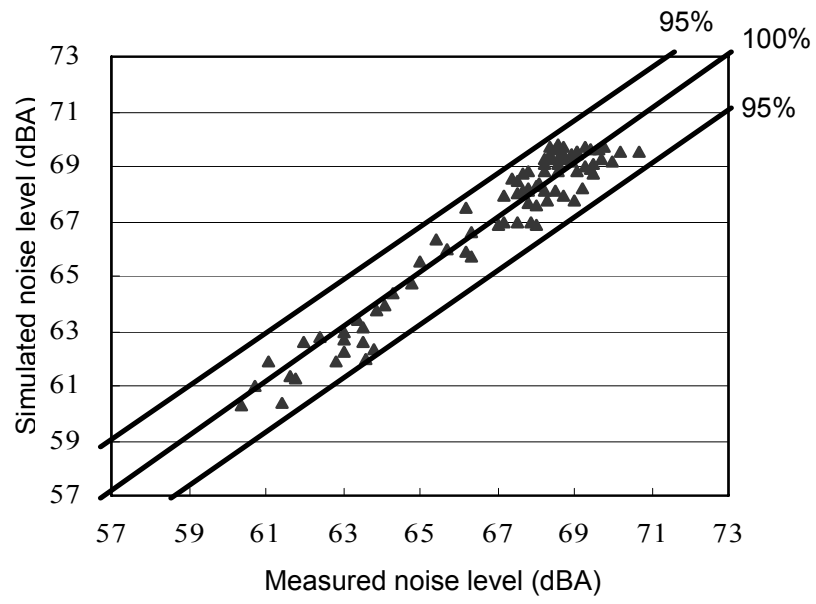

Figure 9. Dimension test of traffic noise level on conventional pavement (before repaved).

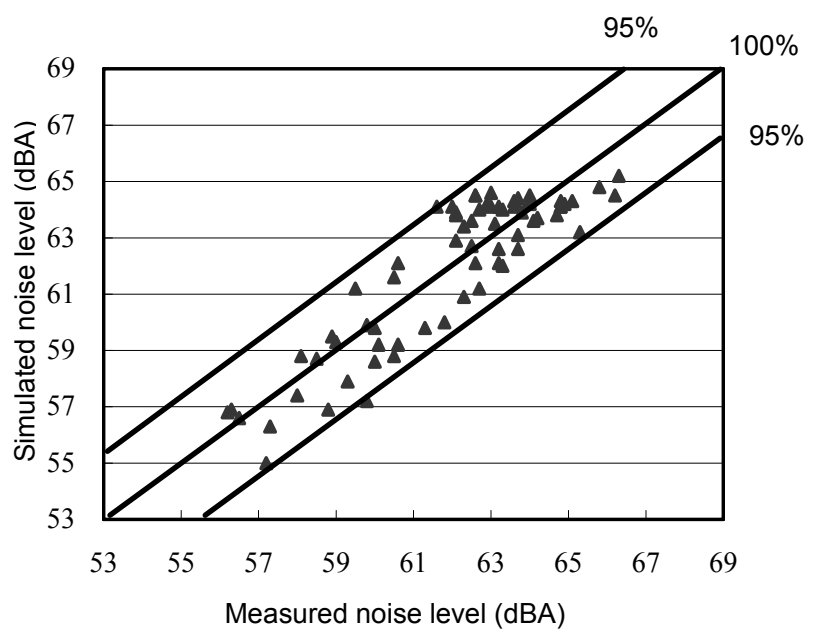

Figure 10. Dimension test of traffic noise level on ARC pavement (after repaved).

Based on the results acquired from the Saskatchewan test road, the traffic noise energy reduction from ARC pavement can be generally expressed as:

$$
E_{(\text {diff })}=\frac{t_{0}}{T}\left(K_{1} \times 10^{7} m_{I}+K_{2} \times 10^{8} m_{I I}+K_{3} \times 10^{8} m_{I I I}\right)
$$

where $K_{1}, K_{2}$ and $K_{3}$ represent the sound intensity reduction 
determined by the tests on the ARC pavement for a light automobile, medium and large truck, respectively. In the present research, $K_{1}=2.99 \times 10^{7}, K_{2}=3.69 \times 10^{8}, K_{3}=4.61 \times$ $10^{8}$.

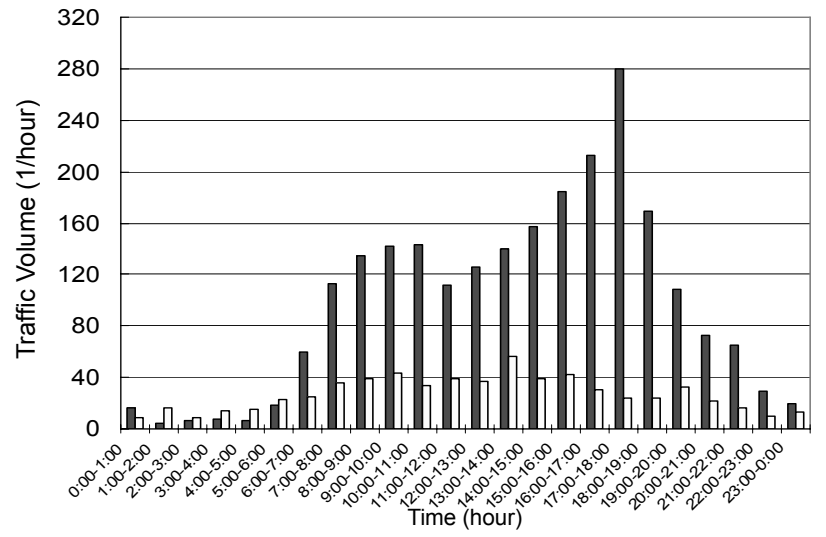

Figure 11. The comparison of the volume of light and heavy vehicles during 24 hours (the data is acquired from northbound on Sep. $\left.19^{\text {th }}, 2005\right)$.

Despite the fact that noise level reductions for various kinds of vehicles are similar, the energy reduction for medium and heavy trucks is ten times larger than that of light vehicles, according to Equation (11). This result demonstrates that noise reduction performance over ARC pavement is more effective for large vehicles. This also agrees with the 24-hour noise level results (Figures 5 and 6). The ARC pavement shows a noise reduction of about $5 \mathrm{~dB}$ at almost any time of the day. During the daytime, light vehicles (Class I) dominate the traffic flow as can be seen from Figure 11. In fact, the traffic volume for light vehicles is about ten times that of larger vehicles as per the traffic flow data collected. However, during the night time, the volume of large vehicles is almost the same as that of light vehicles. Thus, even if the traffic volume and composition at various times of the day are significantly different, noise level reductions are similar during most of the day. As ARC pavement has higher noise reduction ability for heavy vehicles in comparison with that of conventional pavements, the application of ARC pavement on a road that has a high proportion of large vehicles is rational in practice.

\section{Conclusions}

This research aims at an investigation on traffic noise performance of Asphalt Rubber Concrete (ARC) pavement via experimental and numerical approaches. To reveal the characteristics of the traffic noise reduction performance of the ARC pavement, noise data are collected in the field tests on ARC pavement and compared with that of conventional pavements.

Based on the analyses and results generated from the investigation performed for the present research, following are conclusive.
1) The 24-hour time-averaged traffic noise levels $\left(L_{\text {Aeq24h }}\right)$ of ARC pavement show more than $4 \mathrm{dBA}$ lower intensity in comparing with that of the conventional pavement on both the northbound and southbound test roads. This implies that more than 50\% noise energy is reduced with ARC pavement.

2) The 24-hour noise spectral analysis shows that the ARC pavement has a better noise reduction performance in the frequency range between $630 \mathrm{~Hz}$ and $6.3 \mathrm{kHz}$, which is desirable for traffic noise control.

3) The SPB noise test conducted on the Saskatchewan \#11 highway indicates that ARC pavement can reduce the noise emitted by all kinds of vehicles frequently passing on the highway. Especially, ARC pavement has a stronger ability to reduce traffic noise generated by heavy vehicles in comparison with light vehicles.

4) The research results show that equivalent traffic noise level reduction over ARC pavement attributes to the SPB noise reduction for vehicles passing the test section.

5) By analyzing the relationship between hourly traffic noise levels and traffic flow conditions, it is found that traffic noise levels are directly related to the traffic volumes and the ARC pavement can reduce traffic noise levels for most traffic volume conditions especially under the condition of the hourly traffic volume higher than 50 .

6) An approach with establishment of a traffic noise model is developed in the research on the basis of the energetic averaging method for quantitatively simulating traffic noise levels over conventional and ARC pavements. This model can be applied to numerically determine traffic noise levels with the information data of average SPB noise levels, the distance between the noise receiver and the center-line of the traffic, and traffic volume for a given vehicle category. These information data can be easily acquired along the roads. Such an approach for the traffic noise on ARC pavements has not been seen in the current literature.

7) The simulated traffic noise levels are acceptable, within a $95 \%$ confidence for both conventional and ARC pavements.

\section{References}

Ali, S.A. and Tamura, A., (2003). Road Traffic Noise Levels Restrictions and Annoyance in Greater Cairo, Egypt, Appl. Acoust., 64(8), 815-823, doi:10.1016/S0003-682X(03)00031-8.

Bilawchuk, S. (2005). Tire Noise Assessment of Asphalt Rubber Crumb Pavement, Canadian Acoustics, 33, 37-41.

Bruel \& Kjaer (1995 and 1998). Technical Documentation 2238 and 2260 Integrating and Logging Sound Level Meter, Denmark.

Bruel \& Kjaer (2000). Environmental Noise Booklet, Denmark.

Dai, L., Cao, J., Fan, L. and Mobed, N. (2005). Traffic Noise Evaluation and Analysis in Residential Areas of Regina, J. Environ. Inf., 5, 17-25,

Federal Highway Administration (1998). United States of America, FHWA Traffic Noise Model-Technical Manual, Report No. FHWA -PD-96-010 DOT-VNTSC-FHWA-98-2.

Fujiwara, T., Meiarashi, S., Namikawa, Y. and Hasebe, M. (2005). Reduction of Equivalent Continuous A-weighted Sound Pressure Levels by Porous Elastic Road Surfaces, Appl. Acoust., 66(7), 
766-778, doi:10.1016/j.apacoust.2004.12.004.

Ge, J., and Wang, Z. (2003).The Noise Reduction Analyses of Porous Asphalt Pavement Surface. http://www.sesmag.sh.cn/admin/2/doc/ 13772.doc.

Golebiewski, R., Makarewicz, R., Nowak, M. and Preis, A. (2003). Traffic Noise Reduction due to the Porous Road Surface, Appl. Acoust., 64(5), 481-494, doi:10.1016/S0003-682X(02)00124-X.

Lou, Z. and Dai, L. (2006). A study of Traffic Noise Reduction Performance of ARC Pavement Roads and Traffic Flow, ASME International Mechanical Engineering Congress and Exposition, Chicago, IMECE2006-13325.

International Organization for Standardization (1997). Measurement of the Influence of Road Surfaces on Traffic Noise-Part 1, Statistical Pass-by Method 11819-1, Geneva, Switzerland.

International Organization for Standardization (1997). Measurement of the Influence of Road Surfaces on Traffic Noise- Part 2, CloseProximity Method 11819-2, Geneva, Switzerland.

International Organization for Standardization (1995). ISO Standards Handbook: Acoustics, Geneva, Switzerland.

Li, B., Tao, S. and Dawson, R.W. (2002). Evaluation and Analysis of Traffic Noise from the Main Urban Roads in Beijing, Appl. Acoust., 63(10), 1137-1142, doi:10.1016/S0003-682X(02)00024-
5.

Nelson, P.M. (1994). Designing Porous Road Surfaces to reduce traffic noise, available at http://www.trl.co.uk/static/environment/A nnRev97.pdf.

Pamanikabud, P. and Vivitjinda, P. (2002). Noise Prediction for Highway in Thailand, Transport. Res. D-TR E, 7(6), 441-449, doi:10.10 16/S1361-9209(02)00012-3.

Rubber Asphalt Association (2007). Noise Reduction with AsphaltRubber, available at http://www.rubberpavements.org/library/noise reduction.asp.

Ruhala, R. J. (1999). A Study of Tire/Pavement Interaction Noise Using Nearfield Acoustic Holography, UMI Miroform 9940945.

Sacramento County Department of Environmental Review and Assessment and Bollard and Brennan, Inc (2005). Effectiveness of Rubberized Asphalt in Reducing Traffic Noise, available at http:// www.rubberpavements.org/library/sacramento_noise_study/index. html.

Sandberg, U. and Ejsmonnt, J. A. (2002). Tyre/Road Noise Reference Book, NODENA, Poland ISBN 91-631-2610-9.

World Health Organization (WHO) (1999). Guidelines for Community Noise, Geneva, Switzerland. 